The following pages constitute the final, accepted and revised manuscript of the article:

Andreas Lennartsson, Daniel Garwicz, Anders Lindmark and Urban Gullberg

"The proximal promoter of the human cathepsin G gene conferring myeloidspecific expression includes C/EBP, c-myb and PU.1 binding sites”

Gene. 2005 Aug 15;356:193-202.

Publisher: Elsevier

Use of alternative location to go to the published version of the article requires journal subscription.

Alternative location: http://dx.doi.org/10.1016/j.gene.2005.05.004 


\title{
The proximal promoter of the human cathepsin $G$ gene conferring myeloid-specific expression includes C/EBP, c-myb and PU.1 binding sites
}

\author{
Andreas Lennartsson, Daniel Garwicz, Anders Lindmark \\ and *Urban Gullberg \\ Department of Hematology, Lund University, Sweden
}

Key words: serine proteases, granulopoiesis, azurophil, neutrophil, transcriptional regulation

*Corresponding author

Urban Gullberg, Department of Hematology, BMC, C14, S-221 84 Lund, Sweden

Tel: +46-46-222 07 29, Fax: +46-46-18 4493

e-mail: urban.gullberg@hematologi.lu.se 


\section{ABSTRACT}

Cathepsin $\mathrm{G}$ is a hematopoietic serine protease stored in the azurophil granules of neutrophil granulocytes. The mRNA of cathepsin $G$ is transiently expressed during the promyelocyte stage of neutrophil maturation. The protease plays several roles in inflammatory actions of neutrophils, such as bactericidal effects. A human cathepsin $G$ gene fragment of 6 kb directs a promyelocyte-specific expression in transgenic mice, indicating the presence of necessary cisacting elements. However, neither the precise architecture of the promoter, nor the transacting factors responsible for its activation, have been characterized. In the present work, 2.6 kb upstream of the translation start site of the human cathepsin $G$ gene was cloned. When transfected to monoblast-like U937 or to acute promyelocytic leukemia NB4 cells, both expressing endogenous cathepsin $\mathrm{G}$, the initial 360 bp upstream of the translation start were sufficient to direct a strong expression of a luciferase reporter gene. No expression was observed in erythroid K562 control cells. Further deletions revealed three major regulatory regions containing the consensus binding-sites for the transcription factors C/EBP, c-myb and PU.1. Moreover, a GC-rich region, similar to a cis-element in the proteinase 3 promoter, was identified. Direct binding of the trans-factors $\mathrm{C} / \mathrm{EBP} \alpha, \mathrm{C} / \mathrm{EBP} \varepsilon$, c-myb and PU.1 to the promoter was shown by chromatin immunoprecipitation. The functional significance of the cis-elements was verified by site-directed mutagenesis. Mutations of the putative PU.1 site moderately decreased the activity of the promoter in monoblastic U937 cells, but not in promyelocytic NB4 cells. Separate mutations of the putative C/EBP binding site, c-mybbinding site or the GC-rich element resulted in a dramatically reduced transcriptional activity in both cell lines, suggesting cooperation between corresponding trans-factors. 


\section{INTRODUCTION}

The hematopoietic serine proteases constitute a superfamily of enzymes stored in cytoplasmic granules of hematopoietic cells, showing capacity for regulated secretion. Cathepsin G is one of four serine protease family members stored in the azurophil granules of neutrophil granulocytes; the neutrophil serine protease family also includes leukocyte elastase, proteinase 3 and the enzymatically inactive protease homologue azurocidin (Gullberg et al., 1997).

Cathepsin $G$ has been proposed to play several roles in inflammation and host defence. Purified cathepsin $G$ is cytotoxic against, or inhibits the growth of a number of microbes (Shafer and Onunka, 1989) (Shafer et. al., 1991) (Guyonnet et. al., 1991). Elimination of cathepsin $G$ in transgenic mice results in no obvious defects in microbial clearance, but combined deletion of the cathepsin $G$ and leukocyte elastase genes leads to increased susceptibility to bacterial challenges (Tkalcevic et al., 2000). Besides localization in azurophil granules, cathepsin $\mathrm{G}$ is present on the cell surface of neutrophils after degranulation, possibly enhancing chemotactic signaling (Maison et. al., 1991) (Tkalcevic et. al., 2000) (Lomas et al., 1995). Cleavage of several blood clotting factors (e.g. coagulation factor V) and the thrombin receptor, leading to functional activation, are examples of potential extracellular roles of cathepsin G (Allen and Tracy, 1995; Turkington et al., 1986). Uncontrolled proteolytic action of cathepsin G may contribute to tissue destruction seen in various chronic inflammatory disorders, such as allergic rhinitis (Westin et. al., 1999). It has also been proposed that cathepsin $G$ plays a role in tissue remodeling at sites of wounding or tissue injury, by cleavage and inactivation of chemoattractants for neutrophils, such as TNF- $\alpha$ (Scuderi et al., 1991), IL-1 (Hazuda et al., 1990) and IL-8 (Padrines et al., 1994). Consistently, cathepsin G deficient mice have an increased amount of neutrophils at inflammatory sites, which may be due to a high concentration of chemoattractants in the wound fluid (Abbott et al., 1998). 
Neutrophil serine proteases are mainly synthesized during the promyelocytic stage of myeloid differentiation, and after processing into mature enzymes the proteases are stored in azurophil granules as proteolytically active enzymes (Gullberg et. al. 1999) (Cowland and Borregaard, 1999). No sorting motifs for sorting to granules have yet been identified (Gullberg, et. al. 1999). Rather, it has been proposed that differentiation-related transcriptional control of the expression of the cathepsin $G$ gene and other granule components, results in sorting into distinct granule subtypes (Arnljots et al., 1998). Identification of important transactivating factors for the promyelocyte specific expression of elastase and proteinase 3 have been reported (Lutz et al., 2001; Nuchprayoon et al., 1997; Oelgeschlager et al., 1996; Sturrock et al., 1996; Sturrock et al., 2004). These factors include the trancriptional activators PU.1, cmyb, C/EBP and a $40 \mathrm{kDa}$ protein binding to a GC-rich cis-element. In contrast to the elastase and proteinase 3 genes, located on chromosome 19, the cathepsin $G$ gene is located on chromosome 14 in a cluster with the genes encoding the cytotoxic T-cell protease granzyme B and granzyme $H$ (Hanson et al., 1990; Yousef et al 2003). Moreover, cathepsin $G$ seems be expressed somewhat later than elastase and proteinase 3 (Garwicz et. al, 2005). The tissue- and maturation-specific expression of cathepsin $G$ has been linked to a region stretching from $2.5 \mathrm{~kb}$ upstream to $0.8 \mathrm{kbp}$ downstream of the first and last exon, respectively, as judged by its capacity to dictate expression in promyelocytes of transgenic mice (Grisolano et al., 1994). Detailed molecular analysis of the promoter of cathepsin $G$ including transactivating factors is, however, not available. Given the distinct chromosomal localization of cathepsin $G$, as compared to the proteinase 3 and leukocyte elastase genes, we asked whether the proximal promoter shows a similar functional and structural organization. Our results show that within 360 bp upstream of the translation start a proximal promoter is contained, including binding sites for PU.1, c-myb, C/EBP, and a GC-rich element, similar to the promoters of proteinase 3 and leukocyte elastase. Moreover, the C/EBP binding site, c- 
myb binding sites and the GC-rich element show pronounced functional synergy, conferring strong expression from the promoter in myeloid cells.

\section{MATERIALS AND METHODS}

\subsection{Cell culture}

Monoblastic U937, promyelocytic NB4 and erythroid K562 were maintained in RPMI-1640 medium (GIBCO) supplemented with $10 \%$ fetal calf serum (FCS, GIBCO). Exponentially growing cells were used for all experiments.

\subsection{Genomic cloning}

Cloning of human genomic DNA upstream of the transcription start of the cathepsin $G$ gene was performed by PCR, utilizing the human Genomewalker ${ }^{\mathrm{TM}}$-kit (Clontech), according to the manufacturer's recommendations. From published cDNA sequence (Hohn et al., 1989) oligonucleotide downstream primers specific for cathepsin $G$ were synthesized for use in $\begin{array}{lll}\text { nested } & \text { PCR: } & \text { adapt }\end{array}$ 5'-AGGAGAAAGGCCAGCAGAAGC-3' and primer 5’-TCAACTGCTAGCCTTTCCTGAAAGGCTGCCCAGTC-3' (NheI restriction site underlined). Both primers bind to the published cDNA sequence of cathepsin G. All PCR's were performed in a DNA thermal cycler 480 (Perkin Elmer). The resulting PCR products 1.2 $\mathrm{kbp}$ and $2.6 \mathrm{kbp}$, respectively were cloned into luciferase reporter vector pGL3/basic (Promega) using MluI and NheI restriction sites, thus creating the corresponding vectors pGL3/1.2 and pGL3/2.6. Sequencing (Cybergene AB, Huddinge, Sweden) was performed to control the identity, compared with the previously published 5' flanking region of the cathepsin G gene [GeneBank, M59717.1]. As previously reported, a potential TATA-box and 
CAAT-box at position -57 and -97 , respectively, are present in the putative promoter region (numeration from the translation start site) (Hohn et al., 1989).

\subsection{Deletion and mutation constructs}

To create sequential deletions of the promoter region, the nested primer described above was used as downstream primer, together with upstream primers complementary to sequences in the promoter at 807 bp, 564 bp, 360 bp, 300 bp, 250 bp, 200 bp and 150 bp, respectively. The upstream primers included a restriction site for Mlu I. For site directed mutagenesis of potential transcription factor binding sites, oligonucleotide primers including the desired mutations were synthesized and used in a two-step spliced overhang extension (SOE) PCR as described (Gullberg et al., 1992). In the potential binding site for C/EBP, 5’-ATTCT-3’ was changed to 5'-CGGTG-3'; in the PU.1 site, 5'-AGGAG-3' was changed to 5'-TCTTC-3'; in the putative binding site for c-myb, 5'-AGTTT-3' was changed to 5'-CACCG-3'; and in the GC-rich region, 5'-CCCAC-3' was mutated to 5'-AAACA-3'. All PCR's were performed with Pfu-polymerase. After subcloning into pGL3/basic, individual clones were sequenced to verify the creation of the desired mutations. In some constructs 6 or 10 bases were inserted between indicated transcription factor binding sites.

\subsection{Transient transfection}

$15 \mu \mathrm{g}$ of either pGL3/basic vector (negative control), pGL3/control vector (positive control) or pGL3/cathepsin G promoter construct, was mixed with $0.5 \mu \mathrm{g}$ pL/SV40 vector (Promega) and added to U937 or K562 cells in $0.5 \mathrm{ml}$ medium $\left(18 \times 10^{6}\right.$ cells/ml). Expression from pRL/SV40 was used as internal control for transfection efficiency. Transfection was performed using electroporation in a Genepulser II (Biorad) in a $0.4 \mathrm{~cm}$ cuvette, with the 
electrical settings $280 \mathrm{~V} / 960 \mu \mathrm{F}$ at room temperature. Following electroporation, cells were seeded at $0.9 \times 10^{6}$ cells $/ \mathrm{ml}$ medium and incubated for 16 hours at $37^{\circ} \mathrm{C}$ and after that subjected to dual luciferase assay. With NB4 cells, $30 \mu \mathrm{g}$ of the plasmid was used for transfection of $9 \times 10^{6}$ cells/ml at $340 \mathrm{~V} / 960 \mu \mathrm{F}$, and luciferase activity was determined after 10 hours.

\subsection{Dual luciferase assay}

Cell lysis and dual luciferase assay were performed using the Dual Luciferase ${ }^{\circledR}$ reporter assay kit (Promega) according to the manufacturer's recommendations. Briefly, the cells were lysed by treatment with $0.5 \mathrm{ml}$ "passive lysis buffer" (Promega) during continuous shaking for 20 min and repeated vortexing. The light emission of firefly and renilla luciferase of $40 \mu \mathrm{l}$ cell lysate supernatant was quantified in a TD-20/20 luminometer (Turner Biosystems, California, USA). The statistical significance of the difference between mutated/deleted samples and pGL3/360 was estimated with non-parametric Mann-Whitney U test, since the sample number $(n=6)$ was too low to guarantee normal distribution. The statistic analyses were performed with StatView 5.01.

\subsection{Western blotting}

Western blot analysis of PU.1 was performed as previously described (Chylicki et al., 2000). Briefly, $5 \times 10^{6}$ U937 or NB4 cells were harvested, washed and lysed. After electrophoresis, proteins were electrophoretically transferred to a Hybond-P membrane (Amersham Pharmacia Biotech). A rabbit antiserum against PU.1 (sc-352, Santa Cruz) was used as primary antibody (diluted 1:1000) and peroxidase conjugated swine anti-rabbitserum as secondary antibody (P217, DAKO). As equal loading control was antibody against actin (sc-8432, Santa Cruz, diluted 1:5000) was used and peroxidase conjugated goat anti-mouseserum (170-6516, 
Biorad) as secondary antibody. For detection, ECL Plus ${ }^{\mathrm{TM}}$ Western blotting detection system (Amersham Pharmacia Biotech) was used.

\subsection{Chromatin immunoprecipitation (ChIP)}

Chromatin immunoprecipitation (ChIP) was performed using the ChIP assay kit (Upstate Biotechnology), according to the manufacturer's instruction. Briefly, 1 x $10^{6}$ cells were crosslinked with $1 \%$ formaldehyde for $20 \mathrm{~min}$, harvested and sonicated $4 \times 40$ seconds at $100 \%$ power using a UP 50H sonicator (Dr. Hielscher GmbH, Germany). ProteinA-sepharose and 6 $\mu \mathrm{g}$ of antibody were added and incubated overnight at $4^{\circ} \mathrm{C}$; antibodies (Santa Cruz), were against C/EBP $\alpha$ (sc-61x), C/EBPe (sc-158x), c-myb (sc-517x), PU.1 (sc-352x), Sp1 (sc-59x) and cyclin D1 (sc-753). As a negative control was ProteinA-sepharose added, without antibody. After washing and elution procedures according to the manufacturer's instruction, the immunocomplexes were resuspended in $40 \mu \mathrm{l}$ distilled water. In the PCR (33 cycles) 4-10 $\mu \mathrm{l}$ of immunoprecipitated DNA was used as template and primers were as follows: C/EBPsite, forward primer 5'-GGAGTAGAAAACAGGAAGTGGGC-3' and reverse 5'AGATGTGGGTGAGACCAGTGTAATC-3’; PU.1/c-myb/GC-rich-site, forward primer 5’TTGATGGGTTGAGGGTTCTGG-3’ and reverse 5'-CAGTCAGTTGCTGCTGTGCTTC-3'. To verify the ChIP specificity, a control PCR was performed with primers annealing 1-1.5 kbp downstream the translation start, forward primer 5'-CCTGGTGCGAGAAGACTTTGTG-3' reverse 5’-TCCTTTCCTCCTCATTTACACTGG-3’. 


\section{RESULTS}

\subsection{Transcriptional activity studies}

The transcription start of the cathepsin $G$ gene has been determined previously to position -28 (numeration from the translation start site) (Salvesen et al., 1987). To localize cis-acting regions in the promoter responsible for driving expression of cathepsin $G$, we performed transient transfections of pGL3/1.2 and pGL3/2.6 into monoblastic U937 cells or promyelocytic NB4 cells, followed by analysis of luciferase expression as described in "Materials and Methods". Both cell lines show robust expression of endogenous cathepsin $G$, although U937 cells express several fold more cathepsin G mRNA, as determined by RT-real time PCR (data not shown). Transfection of both plasmids resulted in a strong and comparable expression of luciferase Promoter activity of pGL3/1.2 in U937 cells was $37 \%$ of that obtained with the positive control-plasmid containing the viral SV-40 promoter (pGL3/control vector) (Figure 1a), while the activity in NB4 cells was only $14 \%$ (Figure 1b), consistent with the lower expression of endogenous cathepsin $G$ mRNA in these cells (data not shown). The results indicate that critical positive elements for cathepsin $G$ expression are present in the initial $1.2 \mathrm{kbp}$ region, present in both pGL3/1.2 and pGL3/2.6. As control for tissue specificity of the promoter, pGL3/1.2 was transfected to the erythroid human cell line K562 lacking expression of cathepsin $G$ (data not shown). The luciferase activity of pGL3/1.2 in K562 was as low as the background activity obtained with negative control vector pGL3/basic (Figure 1c).

Since pGL3/1.2 showed as strong transcriptional activity as pGL3/2.6, subsequent characterization was limited to the $1.2 \mathrm{kbp}$ region, from which sequentially increasing deletions were made as described in "Materials and Methods". The various PCR-products were cloned into pGL3/basic, creating pGL3/800, pGL3/560, pGL3/360, pGL3/300, pGL3/250, pGL3/200 and pGL3/150, respectively. Progressive deletion of the upstream - 
1,200 to -560 bp sequence resulted in very small changes of transcriptional activity after transfection to U937 cells, while further deletion to -360 bp yielded a twice as strong expression of the luciferase gene as $-1,200$ bp promoter (Figure 1a). In fact, maximal transcriptional activity was seen with pGL3/360 since further deletions strongly suppressed transcriptional activity. Indeed, the promoter activity of pGL3/150 is four-fold lower than the pGL3/360 activity (Figure 1a). In NB4 cells a similar increase in activity was observed upon deletion from -1,200 bp to -360 bp (Figure 1b). However, further deletion to -200 bp did not significantly decrease the promoter activity in NB4 cells. Indeed, deletion to -150 bp was needed to obtain reduced promoter activity (Figure 1b). No part of the promoter showed transcriptional activity in K562 cells (Figure 1c). We conclude that the proximal 360 bp upstream of the translation start contain a proximal promoter directing myeloid-specific expression.

\subsection{Analysis of the proximal promoter by site directed mutagenesis}

By matching the 360 bp promoter sequence to the transcription factor database TRANSFAC (http://motif.genome.jp/) potential cis-elements were identified (Figure 2). Given their involvement in the regulation of other genes expressed at the promyelocyte stage, we chose to study in detail the importance of potential sites for C/EBP, c-myb and PU.1. Thus, the binding sites for C/EBP, c-myb or PU.1 at -335 bp, -175 bp and -46 bp respectively, were mutated in pGL3/360 as described in "Materials and Methods". The modified constructs were transfected to U937 and NB4 cells, followed by measurements of the luciferase activity. Moreover, the GC-rich element at -86 bp was mutated, since a similar sequence is important for expression of proteinase 3 and leukocyte elastase genes (Sturrock et al., 1996) (Sturrock et al., 2004). Mutation of the C/EBP or c-myb binding sites resulted in a strong inhibition of the transcriptional activity in U937 cells, five- and eleven-fold respectively. Moreover, a 
fourteen-fold reduction was caused by mutation of the GC-rich element (Figure 3a). Mutation of the PU.1 site affected expression more moderately, resulting in approximately 50\% reduction (Figure 3a). In NB4 cells, similar effects were observed although the relative reduction after mutation of the C/EBP, c-myb site or the GC-rich element was only two-fold, compared to the dramatic decrease in U-937 cells. Furthermore, mutation of the PU.1 site did not affect the transcriptional activity in NB4 cells (Figure 3b). We conclude that the C/EBP and c-myb sites, as well as the GC-rich element, are critical for the transcriptional activity of cathepsin $G$ proximal promoter. Moreover, the potential PU.1 site contributes to promoter activity in monocytic U937 cells, but not in myeloid NB4 cells, suggesting higher PU.1activity in U937 cells. Western blotting of PU.1 indeed showed a higher level of PU.1 in U937 cells (Figure 4), consistent with a role of PU.1 as an important transcription factor for high expression of cathepsin $G$.

\subsection{Binding of C/EBP, c-myb and PU.1 to cathepsin $G$ promoter in vivo}

Our results indicate direct binding of C/EBP, c-myb and PU.1 to the promoter of cathepsin $G$. In addition, Sp1 is a candidate for binding to the GC-rich site (Imataka et al., 1992). To demonstrate direct binding in vivo, we performed chromatin immuno-precipitation (ChIP) as described in "Materials and Methods". After crosslinking of proteins to DNA in U937 cells, the DNA was sonicated. Immunoprecipitations with antibodies to $\mathrm{C} / \mathrm{EBP} \alpha, \mathrm{C} / \mathrm{EBP} \varepsilon, \mathrm{Sp} 1$, PU.1 or c-myb were performed, followed by PCR-amplification of the promoter-region with specific primers. As negative controls, immunoprecipitation with anti-cyclin D1 or addition of only Protein-A sepharose were performed. As shown in Figure 5a and 5b, immunoprecipitation with antibodies to $\mathrm{C} / \mathrm{EBP} \alpha, \mathrm{C} / \mathrm{EBP} \varepsilon$, PU.1 or c-myb resulted in amplification of the promoter region, while immunoprecipitation with anti-Sp1 and anticyclin D1 did not. These results demonstrate direct binding of $\mathrm{C} / \mathrm{EBP} \alpha, \mathrm{C} / \mathrm{EBP} \varepsilon, \mathrm{c}-\mathrm{myb}$ and 
PU.1 to the promoter in U937 cells. Moreover, PCR amplification of immunoprecipitated DNA with a primer pair annealing 1-1.5 kbp downstream the C/EBP, c-myb and PU.1 ciselements did only result in equal amplification at the background level (Figure 5c), indicating DNA shearing sufficient for specific immunoprecipitation.

\subsection{Characterization of co-operative activation of the cathepsin $G$ promoter}

The dramatic effect on transcriptional activity by mutation of the c-myb-site, C/EBP-site or the GC-rich element (Figure 3a), suggested that these cis-elements with corresponding transcription factors functionally interact with each other. Therefore, minor changes in the architecture of the promoter should disrupt or reduce transcriptional activity. To investigate this issue, we introduced small insertions between the C/EBP and the c-myb site, or between the c-myb site and the GC-rich element. Six bp or ten bp insertions were introduced to create half- or full helical turns of the DNA-helix, respectively (Idres et al., 2001). The reporter constructs were transfected to U937 cells, since the previously described mutations of the binding sites had the most pronounced effect in this cell line (Figure 3). A 6 bp insertion between the C/EBP and c-myb sites slightly inhibited the promoter activity, while a $10 \mathrm{bp}$ insertion fully restored the activity (Figure 6). Although observed differences in activity did not reach statistical significance, the results are consistent with the notion that an interaction between transcription factors binding to the C/EBP and c-myb sites are important for promoter activity and that correct three-dimensional positioning of the turn of the DNA-helix is necessary for full functional interaction. A 6 bp insertion between the c-myb and GC-rich element did not significantly reduce transcriptional activity, while a 10 bp insertion clearly reduced promoter activity almost two-fold (Figure 6), further suggesting that an interaction between transcriptions factors is dependent on correct distance to each other. 


\section{DISCUSSION}

The core promoter of a gene is positioned immediately adjacent to and upstream of the transcription start. The transcription start of the cathepsin $G$ gene is located 28 bp upstream of the translation start (Salvesen et al 1987). The core promoter binds RNA polymerase II and its accessory factors and directs the transcription to the start site. Several myeloid genes, including those encoding CD18 and CD11b receptor, do not contain a TATA-box (Rosmarin et al., 1992) (Pahl et al., 1991). The azurophil granule proteins bactericidal/permeability increasing protein (BPI) (Lennartsson et al., 2003), proteinase 3 (Sturrock et al., 1996) and cathepsin G (Hohn et al., 1989), differ in that respect, since they do contain a TATA-box. However, the functionality of the TATA-boxes in these genes remains to be demonstrated. The regulatory promoter of a gene conferring tissue specific expression is typically within the first 500 bp upstream of the transcription start. In the present investigation, functional regulatory elements of the promoter of cathepsin $G$ have been defined.

By screening the 5' flanking region of the cathepsin $G$ gene (2.6 kbp upstream of the transcription start) for regulatory cis-elements, we found that the initial 360 bp were sufficient for maximal tissue specific expression. This conclusion is based on the findings of maximal promoter activity in U937 and NB4 cells, both with strong expression of endogenous cathepsin $G$, while the promoter was completely inactive in K562 cells, showing no expression of cathepsin G. Further deletion studies revealed important cis-elements in the regions 360-300 bp and 200-150 bp. In the identified region potential C/EBP and a c-myb binding sites are located at positions -335 bp and -175 bp, respectively. Our finding that mutations of these sites strongly decreased the transcriptional activity of the promoter confirms their importance. Five basepairs in each cis-element were mutated to give a strong modulation of the site without interfering with adjacent elements. C/EBP and c-myb regulate also other myeloid proteins, including the serine proteases proteinase 3 and leukocyte elastase 
(Nuchprayoon et al., 1997; Tsutsumi-Ishii et al., 2000) (Oelgeschlager et al., 1996; Sturrock et al., 1996), which makes them likely to be involved also in the regulation of cathepsin G. Indeed, direct binding of $\mathrm{C} / \mathrm{EBP} \alpha, \mathrm{C} / \mathrm{EBP} \varepsilon$ and c-myb, as shown by ChIP-analysis, confirms their role as transactivators of cathepsin $G$ gene expression. In addition to the identified elements, distant cis-elements important for full physiological promoter activation may be located further up- or downstream of the identified promoter. Gene expression of both proteinase 3 and leukocyte elastase have been shown to be regulated by a distant enhancer element, binding Sp1 (Nuchprayoon et. al. 1999). Similar distant activating cis-elements can not be excluded for cathepsin $G$.

The transcription factor binding to the GC-rich element was not precipitated with antibody against Sp1 and the identity of this trans-acting factor is therefore not clarified. Both proteinase 3 and leukocyte elastase promoters contain a similar GC-rich element, to which an unknown 40kDa protein is bound (Sturrock et al., 1996) (Sturrock et al., 2004). It is therefore likely that a common $40 \mathrm{kDa}$ protein binds to the GC-rich element in the proteinase 3, elastase and cathepsin $G$ promoter. Moreover, all three serine protease gene promoters contain a functional PU.1 binding site just downstream to the GC-rich element, indicating a similar promoter organisation.

The dramatic reduction of promoter activity after mutation of either the c-myb, C/EBP or GCrich element suggests that these cis-elements and corresponding transcription factors cooperatively regulate promoter activity. This notion is supported by the decrease of transcriptional activity as a result of small insertions in the DNA sequence between the ciselements. In order not to disturb the binding of transcription factors, the insertions were made at a 19-124 bp distance from the cis-elements. Evidence for a direct protein interaction between transcription factors remains, however, to be demonstrated. 
PU.1 is a myeloid master regulator involved in the regulation of many myeloid specific genes, and PU.1 is together with $\mathrm{C} / \mathrm{EBP} \alpha$ essential for differentiation into the granulocytic lineage (Sturrock et al., 2004) (Borregaard et al., 2001). Paradoxically, C/EBP $\alpha$ can antagonize PU.1 in the lineage choice into neutrophilic differentiation, whereas PU.1 activity is more important for monocytic differentiation (Reddy et. al., 2002). However, it has been shown that antagonistic relationships can become co-operative under certain circumstances, such as for PU.1 and GATA-2 (Walsh et. al., 2002). Our results indicate that C/EBP and PU.1 cooperate in transcriptional activation of cathepsin G, as judged by the twofold reduction of transcriptional activity in monoblastic U937 cells after mutation of the PU.1 site. In promyelocytic NB4 cells, on the other hand, no effect on transcriptional activity of the PU.1 binding site mutation was seen, indicating that PU.1 activity is higher in U937 cells. This notion is supported by the finding of higher levels of PU.1 protein in U937 cells, as compared to NB4 cells. However, the protein level and transcriptional activity do not necessarily correlate, since PU.1 activity is also regulated post-transcriptionally (Marden et. al., 2003).

In conclusion, in spite of distinct chromosomal localization, the proximal promoter of the cathepsin $G$ gene shows a similar functional and structural organization as those of the proteinase 3 and leukocyte elastase genes. We demonstrate that the promoter of cathepsin $G$ is co-operatively activated by $\mathrm{C} / \mathrm{EBP} \alpha, \mathrm{C} / \mathrm{EBP} \varepsilon, \mathrm{c}-\mathrm{myb}$ and a transcription factor binding to a GC-rich element. In addition, PU.1-binding is important for strong transcription of cathepsin $G$, particularly in monocytic cells. 


\section{ACKNOWLEDGEMENTS:}

The authors would like to thank Karin Arvidsson Green, Tove Ullmark and Johan Wieslander for important experimental contributions and Sophie Ohlsson for help with statistical analyses. This work was supported by grants from the Swedish Foundation for Strategic Research, the Swedish Cancer Society, the Swedish Research Council (project \#11546), the Swedish Childhood Cancer Foundation, the Georg Danielsson Foundation, the Gunnar Nilsson Cancer Foundation, the Greta and Johan Kock Foundation, the John and Augusta Persson Foundation, the Alfred Österlund Foundation, the Lundberg Foundation, the Swedish Society for Medical Research (SSMF), the Swedish Society of Medicine, Funds of Lund University Hospital, and Government funding for clinical research. 


\section{REFERENCES:}

Abbott, R. E., Corral, C. J., MacIvor, D. M., Lin, X., Ley, T. J. and Mustoe, T. A. (1998).

Augmented inflammatory responses and altered wound healing in cathepsin G-deficient mice Arch Surg 133, 1002-6.

Allen, D. H. and Tracy, P. B. (1995). Human coagulation factor V is activated to the functional cofactor by elastase and cathepsin G expressed at the monocyte surface, J Biol Chem 270, 1408-15.

Arnljots, K., Sørensen, O., Lollike, K. and Borregaard, N. (1998). Timing, targeting and sorting of azurophil granule proteins in human myeloid cells, Leukemia 12, 1789-95.

Borregaard, N., Theilgaard-Mönch, K., Sørensen, O. E. and Cowland, J. B. (2001).

Regulation of human neutrophil granule protein expression, Curr Opin Hematol 8, 23-7.

Chylicki, K., Ehinger, M., Svedberg, H. and Gullberg, U. (2000). Characterization of the molecular mechanisms for p53-mediated differentiation, Cell Growth Differ 11, 561-71.

Cowland, J. B. and Borregaard, N. (1999). The individual regulation of granule protein mRNA levels during neutrophil maturation explains the heterogeneity of neutrophil granules, J Leukoc Biol 66, 989-95.

Garwicz D., Lennartsson A., Jacobsen SE., Gullberg U. and Lindmark A. (2005).

Biosynthetic profiles of neutrophil serine proteases in a human bone marrow-derived cellular myeloid differentiation model. Haematologica 90, 38-44.

Grisolano, J. L., Sclar, G. M. and Ley, T. J. (1994). Early myeloid cell-specific expression of the human cathepsin G gene in transgenic mice, Proc Natl Acad Sci U S A 91, 8989-93.

Gullberg, U., Andersson, E., Garwicz, D., Lindmark, A. and Olsson, I. (1997). Biosynthesis, processing and sorting of neutrophil proteins: insight into neutrophil granule development, Eur J Haematol 58, 137-53.

Gullberg, U., Lantz, M., Lindvall, L., Olsson, I. and Himmler, A. (1992). Involvement of an Asn/Val cleavage site in the production of a soluble form of a human tumor necrosis factor (TNF) receptor. Site-directed mutagenesis of a putative cleavage site in the p55 TNF receptor chain, Eur J Cell Biol 58, 307-12.

Gullberg U, Bengtsson N, Bulow E, Garwicz D, Lindmark A and Olsson I. (1999). Processing and targeting of granule protein in Human Neutrophils, J Immunol Methods. 232, 201-10 
Guyonnet, V., Johnson, J. K., Bangalore, N., Travis, J. and Long, P. L. (1991). In vitro activity of the human neutrophil cathepsin G on Eimeria tenella sporozoites, J Parasitol 77, 775-9.

Hanson, R. D., Connolly, N. L., Burnett, D., Campbell, E. J., Senior, R. M. and Ley, T. J. (1990). Developmental regulation of the human cathepsin G gene in myelomonocytic cells, J Biol Chem 265, 1524-30.

Hazuda, D. J., Strickler, J., Kueppers, F., Simon, P. L. and Young, P. R. (1990). Processing of precursor interleukin 1 beta and inflammatory disease, J Biol Chem 265, 6318-22.

Hohn, P. A., Popescu, N. C., Hanson, R. D., Salvesen, G. and Ley, T. J. (1989). Genomic organization and chromosomal localization of the human cathepsin G gene, J Biol Chem 264, 13412-9.

Hsu, H. C., Tsai, W. H., Chen, P. G., Hsu, M. L., Ho, C. K. and Wang, S. Y. (1999). In vitro effect of granulocyte-colony stimulating factor and all-trans retinoic acid on the expression of inflammatory cytokines and adhesion molecules in acute promyelocytic leukemic cells, Eur J Haematol 63, 11-8.

Idres, N., Benoit, G., Flexor, M. A., Lanotte, M. and Chabot, G. G. (2001). Granulocytic differentiation of human NB4 promyelocytic leukemia cells induced by all-trans retinoic acid metabolites, Cancer Res 61, 700-5.

Imataka, H., Sogawa, K., Yasumoto, K., Kikuchi, Y., Sasano, K., Kobayashi, A., Hayami, M. and Fujii-Kuriyama, Y. (1992). Two regulatory proteins that bind to the basic transcription element (BTE), a GC box sequence in the promoter region of the rat P-4501A1 gene, Embo J $11,3663-71$.

Lennartsson, A., Pieters, K., Ullmark, T., Vidovic, K. and Gullberg, U. (2003). AML-1, PU.1, and Sp3 regulate expression of human bactericidal/permeability-increasing protein, Biochem Biophys Res Commun 311, 853-63.

Lomas, D. A., Stone, S. R., Llewellyn-Jones, C., Keogan, M. T., Wang, Z. M., Rubin, H., Carrell, R. W. and Stockley, R. A. (1995). The control of neutrophil chemotaxis by inhibitors of cathepsin G and chymotrypsin, J Biol Chem 270, 23437-43.

Lutz, P. G., Houzel-Charavel, A., Moog-Lutz, C. and Cayre, Y. E. (2001). Myeloblastin is an Myb target gene: mechanisms of regulation in myeloid leukemia cells growth-arrested by retinoic acid, Blood 97, 2449-56. 
Maison, C. M., Villiers, C. L. and Colomb, M. G. (1991). Proteolysis of C3 on U937 cell plasma membranes. Purification of cathepsin G, J Immunol 147, 921-6.

Marden, C. M., Stefanidis, D., Cunninghame-Graham, D. S. and Casimir, C. M. (2003). Differentiation-dependent up-regulation of p47(phox) gene transcription is associated with changes in PU.1 phosphorylation and increased binding affinity, Biochem Biophys Res Commun 305, 193-202.

Nuchprayoon, I., Simkevich, C. P., Luo, M., Friedman, A. D. and Rosmarin, A. G. (1997). GABP cooperates with c-Myb and C/EBP to activate the neutrophil elastase promoter, Blood 89, 4546-54.

Nuchprayoon I., Shang J., Simkevich C., Luo M., Rosmarin A. G. and Friedman A. D. (1999). An enhancer located between neutrophil elastase and proteinase 3 promoters is activated by Sp1 and an Ets factor, J Biol Chem, 274, 1085-91.

Oelgeschlager, M., Nuchprayoon, I., Luscher, B. and Friedman, A. D. (1996). C/EBP, c-Myb, and PU.1 cooperate to regulate the neutrophil elastase promoter, Mol Cell Biol 16, 4717-25. Padrines, M., Wolf, M., Walz, A. and Baggiolini, M. (1994). Interleukin-8 processing by neutrophil elastase, cathepsin G and proteinase-3, FEBS Lett 352, 231-5.

Pahl, H. L., Burn, T. C. and Tenen, D. G. (1991). Optimization of transient transfection into human myeloid cell lines using a luciferase reporter gene, Exp Hematol 19, 1038-41.

Park, D. J., Chumakov, A. M., Vuong, P. T., Chih, D. Y., Gombart, A. F., Miller, W. H., Jr. and Koeffler, H. P. (1999). CCAAT/enhancer binding protein epsilon is a potential retinoid target gene in acute promyelocytic leukemia treatment, J Clin Invest 103, 1399-408.

Reddy, V. A., Iwama, A., Iotzova, G., Schulz, M., Elsasser, A., Vangala, R. K., Tenen, D. G., Hiddemann, W. and Behre, G. (2002). Granulocyte inducer C/EBPalpha inactivates the myeloid master regulator PU.1: possible role in lineage commitment decisions, Blood 100, 483-90.

Rosmarin, A. G., Levy, R. and Tenen, D. G. (1992). Cloning and analysis of the CD18 promoter, Blood 79, 2598-604.

Salvesen, G., Farley, D., Shuman, J., Przybyla, A., Reilly, C. and Travis, J. (1987). Molecular cloning of human cathepsin G: structural similarity to mast cell and cytotoxic T lymphocyte proteinases, Biochemistry 26, 2289-93. 
Scuderi, P., Nez, P. A., Duerr, M. L., Wong, B. J. and Valdez, C. M. (1991). Cathepsin-G and leukocyte elastase inactivate human tumor necrosis factor and lymphotoxin, Cell Immunol 135, 299-313.

Seale, J., Delva, L., Renesto, P., Balitrand, N., Dombret, H., Scrobohaci, M. L., Degos, L., Paul, P. and Chomienne, C. (1996). All-trans retinoic acid rapidly decreases cathepsin G synthesis and mRNA expression in acute promyelocytic leukemia, Leukemia 10, 95-101. Shafer, W. M. and Onunka, V. C. (1989). Mechanism of staphylococcal resistance to nonoxidative antimicrobial action of neutrophils: importance of $\mathrm{pH}$ and ionic strength in determining the bactericidal action of cathepsin G, J Gen Microbiol 135, 825-30.

Shafer, W. M., Pohl, J., Onunka, V. C., Bangalore, N. and Travis, J. (1991). Human lysosomal cathepsin $\mathrm{G}$ and granzyme B share a functionally conserved broad spectrum antibacterial peptide, J Biol Chem 266, 112-6.

Sturrock, A., Franklin, K. F. and Hoidal, J. R. (1996). Human proteinase-3 expression is regulated by PU.1 in conjunction with a cytidine-rich element, J Biol Chem 271, 32392-402. Sturrock, A., Franklin, K. F., Norman, K. and Hoidal, J. R. (2004). Human leukocyte elastase gene expression is regulated by PU.1 in conjunction with closely associated cytidine-rich and Myb binding sites, Biochim Biophys Acta 1676, 104-11.

Tkalcevic, J., Novelli, M., Phylactides, M., Iredale, J. P., Segal, A. W. and Roes, J. (2000). Impaired immunity and enhanced resistance to endotoxin in the absence of neutrophil elastase and cathepsin G, Immunity 12, 201-10.

Tsutsumi-Ishii, Y., Hasebe, T. and Nagaoka, I. (2000). Role of CCAAT/enhancer-binding protein site in transcription of human neutrophil peptide-1 and -3 defensin genes, J Immunol 164, 3264-73.

Turkington, P. T., Blumsom, N. L. and Elmore, D. T. (1986). The degradation of bovine and human prothrombin by human polymorphonuclear leukocyte cathepsin G, Thromb Res 44, 339-45.

Walsh, J. C., DeKoter, R. P., Lee, H. J., Smith, E. D., Lancki, D. W., Gurish, M. F., Friend, D. S., Stevens, R. L., Anastasi, J. and Singh, H. (2002). Cooperative and antagonistic interplay between PU.1 and GATA-2 in the specification of myeloid cell fates, Immunity 17, 665-76. 
Westin, U., Lundberg, E., Wihl, J. A. and Ohlsson, K. (1999). The effect of immediatehypersensitivity reactions on the level of SLPI, granulo cyte elastase, alpha1-antitrypsin, and albumin in nasal secretions, by the method of unilateral antigen challenge, Allergy 54, 85764.

Wong, E. T., Jenne, D. E., Zimmer, M., Porter, S. D. and Gilks, C. B. (1999). Changes in chromatin organization at the neutrophil elastase locus associated with myeloid cell differentiation, Blood 94, 3730-6.

Yousef G, Kopolovic M, Elliott M. and Diamandis., (2003). Genomic overview of serine proteases, Biochem Biophys Res Commun, 305, 28-36. 


\section{FIGURE LEGENDS}

Figure 1. Transcriptional activity after progressive 5'deletions of the cathepsin G promoter. Transfection of indicated deletion constructs to (a) U937, (b) NB4, and (c) K562 cells. Shown is luciferase activity normalized to the activity obtained with pGL3/360 plasmid. The error bars represent the standard error of the mean (SEM) $(n=3)$. Statistically significant differences (P-value $<0.05$ ) from pGL3/360 luciferase activity are labeled with an asterisk (*).

\section{Figure 2. The proximal promoter region of cathepsin G gene with putative cis-regulatory}

elements boxed. The G upstream of the translation ATG start codon is designated -1 .

Figure 3. Transcriptional activity after site directed mutagenesis of putative cis-elements in the cathepsin G promoter. Transfection of pGL3/360 plasmid containing indicated mutations into (a) U937 and (b) NB4 cells. Shown is luciferase activity normalized to the activity obtained with non-mutated pGL3/360 plasmid. The error bars represent SEM $(n=3)$. Statistical significant differences (P-value <0.05) from pGL3/360 luciferase activity are labeled with an asterisk (*).

Figure 4. PU.1 protein in NB4 and U937 cells. Cell lysates were subjected to SDS-PAGE and immunoblot analysis using a polyclonal antiserum against PU.1. Immunoblotting with antibody against actin was used as equal loading control. 
Figure 5. Binding of $\mathrm{C} / \mathrm{EBP} \alpha, \mathrm{C} / \mathrm{EBP} \varepsilon, c-m y b$ and PU.1 to the cathepsin $G$ promoter in U937 cells. Chromatin immunoprecipitation was performed, after which PCR-amplification of the cathepsin $G$ promoter was performed. Specific antibodies against $\mathrm{C} / \mathrm{EBP} \alpha$ or $\mathrm{C} / \mathrm{EBP} \varepsilon$ (a) and c-myb, PU.1, or Sp1 (b) were used in the immunoprecipitation. As negative controls antibodies against cyclin D1 or no antibodies (neg) were used in the immunoprecipitation. As a control for sufficient DNA shearing and immunoprecipitation specificity, primers pair annealing 1-1.5 kbp downstream the C/EBP, c-myb and PU.1 binding sites were used in the PCR-amplification (c).

Figure 6. Transcriptional activity after small insertions between putative cis-elements in the cathepsin $\boldsymbol{G}$ promoter. Six bp or 10 bp insertions were made between cis-elements in the pGL3/360 plasmid, as indicated, to induce disruption and re-establishment, respectively, of the DNA-strand helical phasing. After transfection of the mutated pGL3/360 plasmids to U937 cells, luciferase assay was performed. The luciferase activity normalized to the activity obtained with non-mutated pGL3/360 plasmid is shown. The error bars represent the SEM $(\mathrm{n}=3)$. Statistical significant differences $(\mathrm{P}-\mathrm{value}<0.05)$ from pGL3/360 luciferase activity are labeled with an asterisk (*). 
Figure 1a.

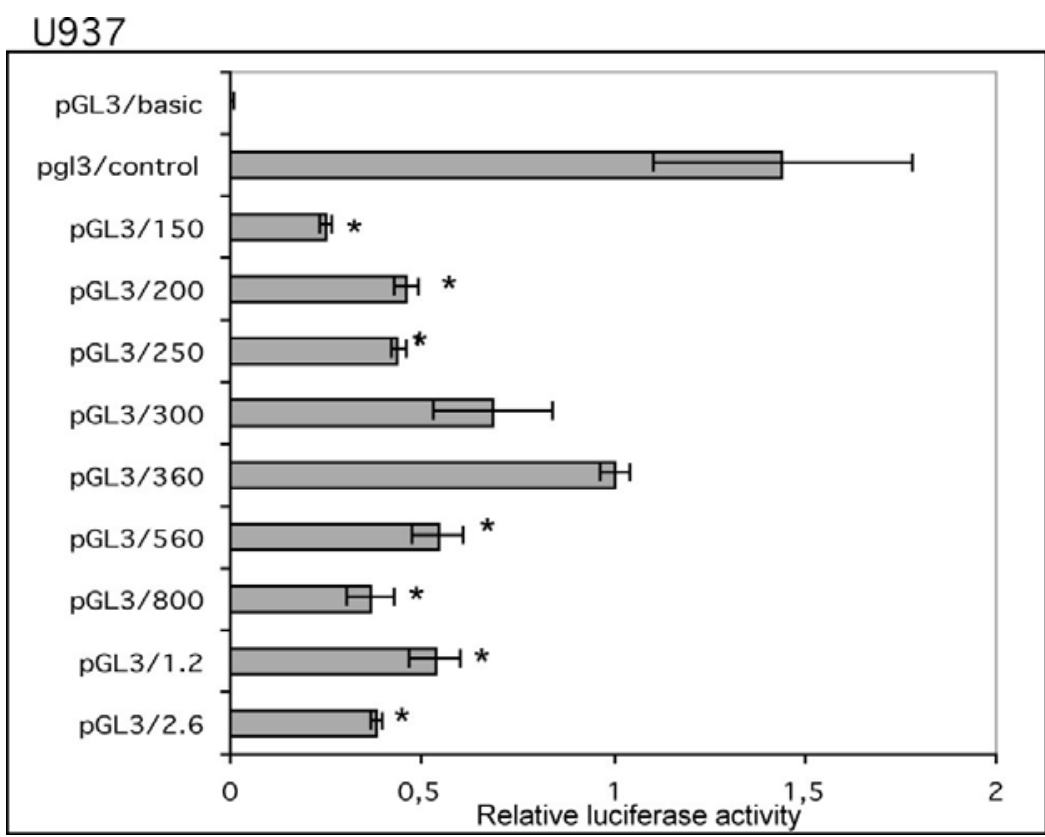

\section{Figure $1 b$.}

NB4

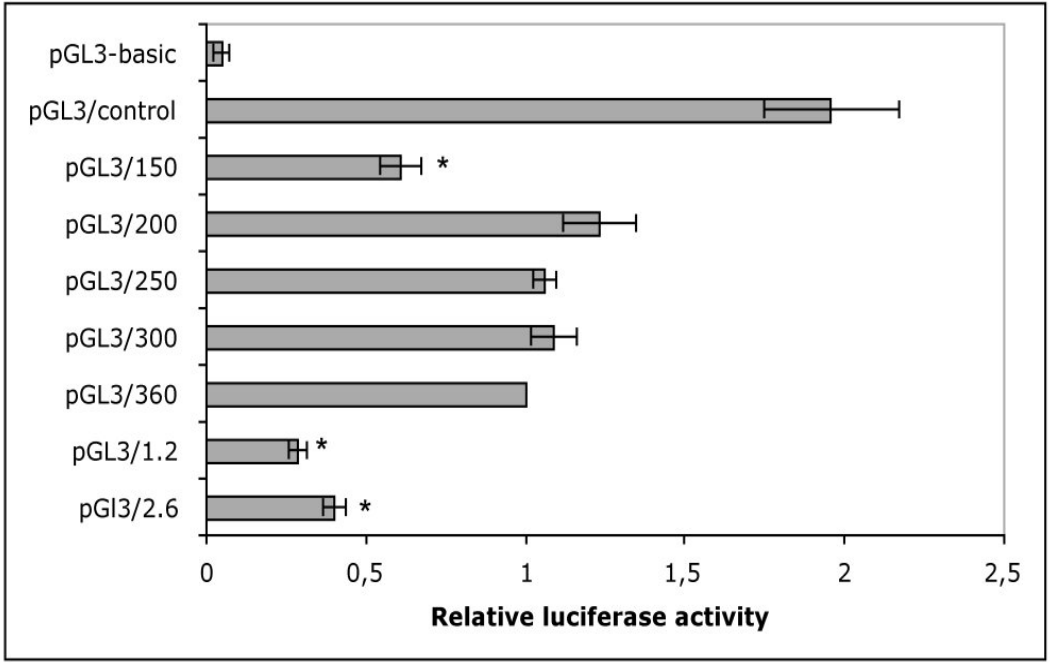




\section{Figure 1c.}

\section{K562}

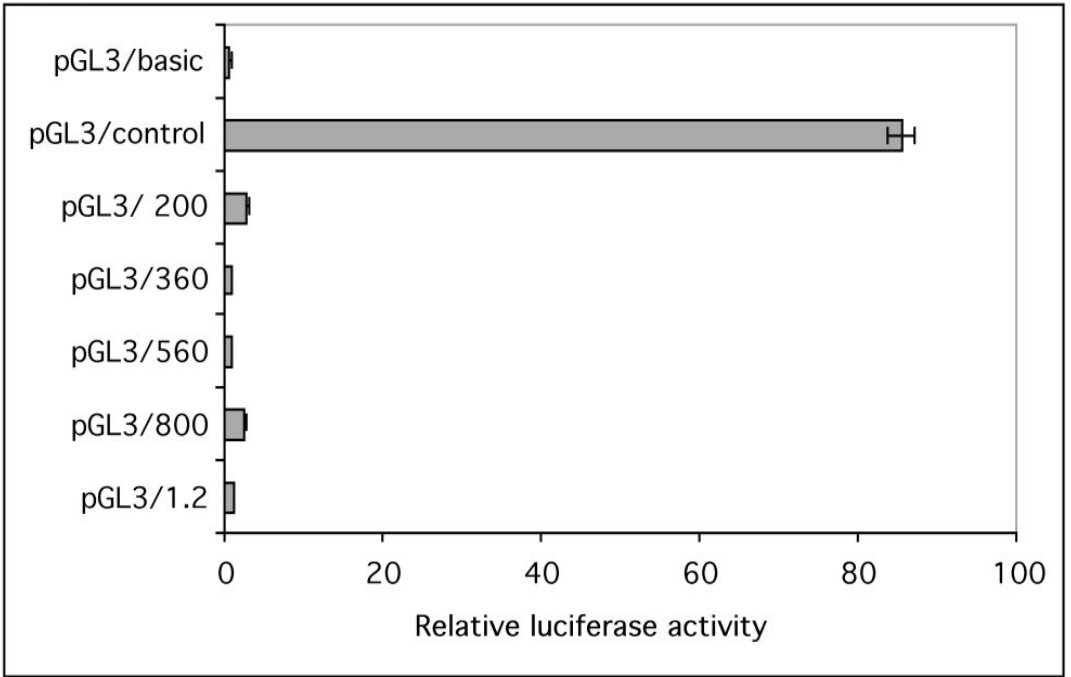

\section{Figure 2.}

- 400-TGATCTAATC CCAGAGGAGT AGAAAACAGG AAGTGGGCAG CTGCCAGCTT TCTTGCTTTG $\mathrm{C} / \mathrm{EBP}$

- 340-CTGGA GTATT CTGGAATTT G ATGGGTTGAG GGTTCTGGAC ACAATGCCCC AAGCCCCTTC

- 280-CTTGTTGTGC TGGGTTCCTA TTTCTGCTCT CGGCACTGAC TTAGCAGCTG CTCAAGAGCT

C-myb

- 220- CACTATGTTG GCTTGGATtA CACTGGTCTC ACCCACATCT CCG GCAGTT TGTGGGCAAAC

- 160-CTCCTGAGCA GCCTTGGGTG ATGAAACCTT TCATGGTAGC AGGAGAATGG GACTGTGAAT

$\begin{array}{cccc}\text { CAAT-box } & \text { GC-rich element } & \text { TATA-box } & \text { PU.1 } \\ \text { - } & \text { 100-TCT CAATCCC CTGT CCCCACC CCTTCCTTC CTCTCTCAGG GCQTTAAAGT CT AGGAGGAG }\end{array}$

Transcription start

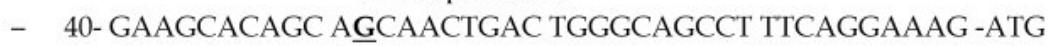


Figure $3 a$.

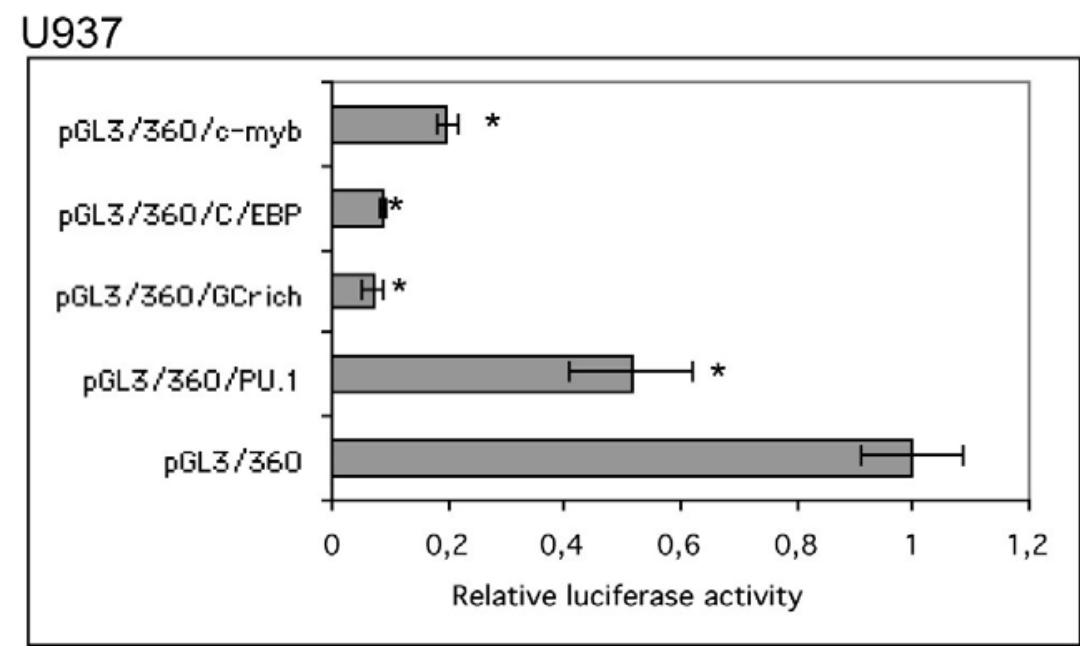

Figure $3 b$.

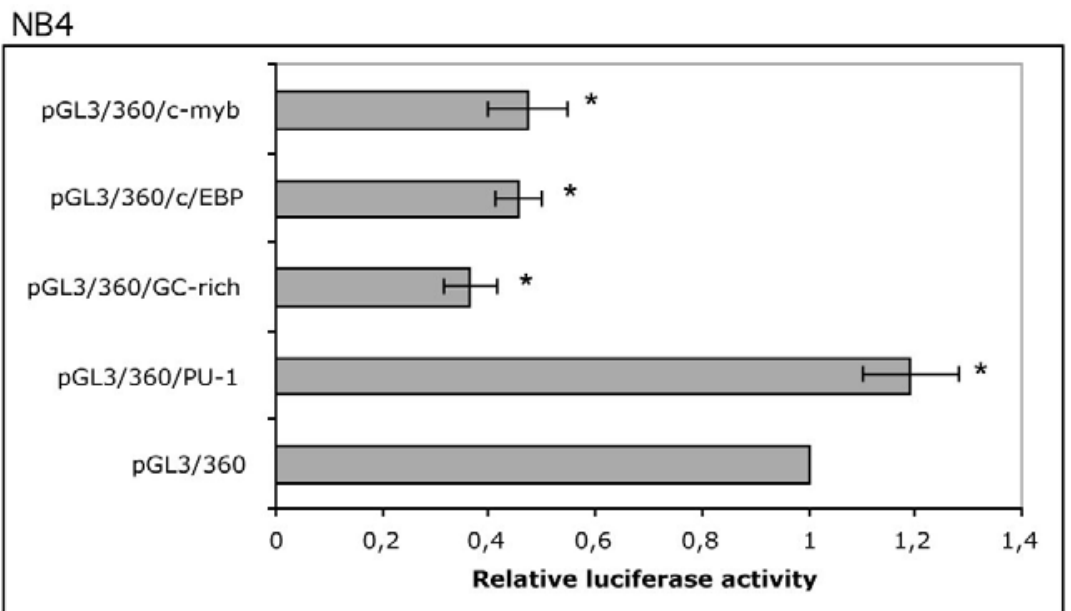




\section{Figure 4.}

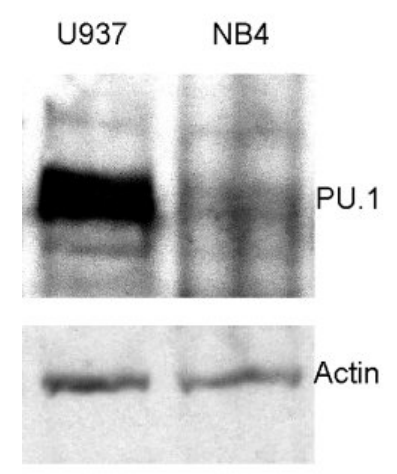

Figure 5a.

$\mathrm{C} / \mathrm{EBP} \alpha \mathrm{C} / \mathrm{EBP \varepsilon}$ cyclinD1 neg

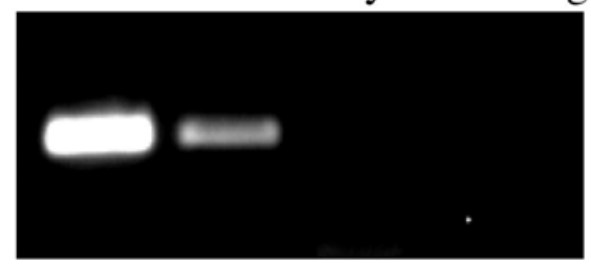

Figure $5 b$.

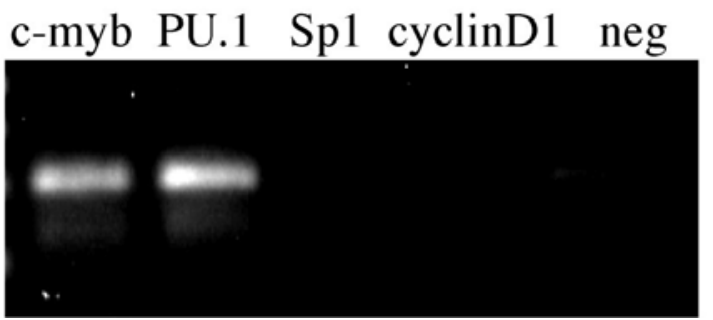

Figure 5c.

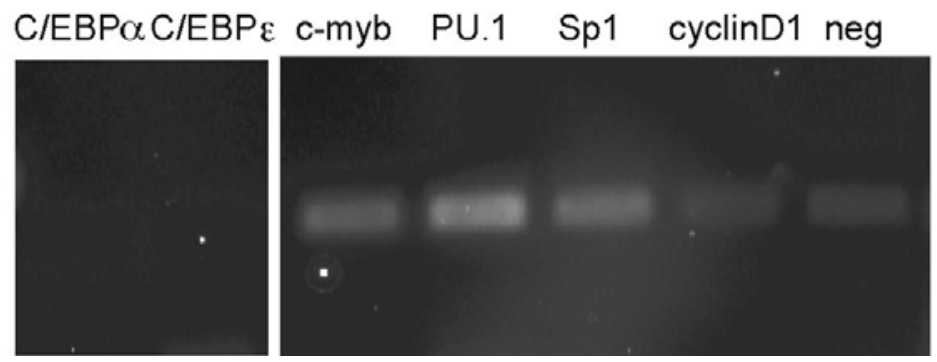


Figure 6.

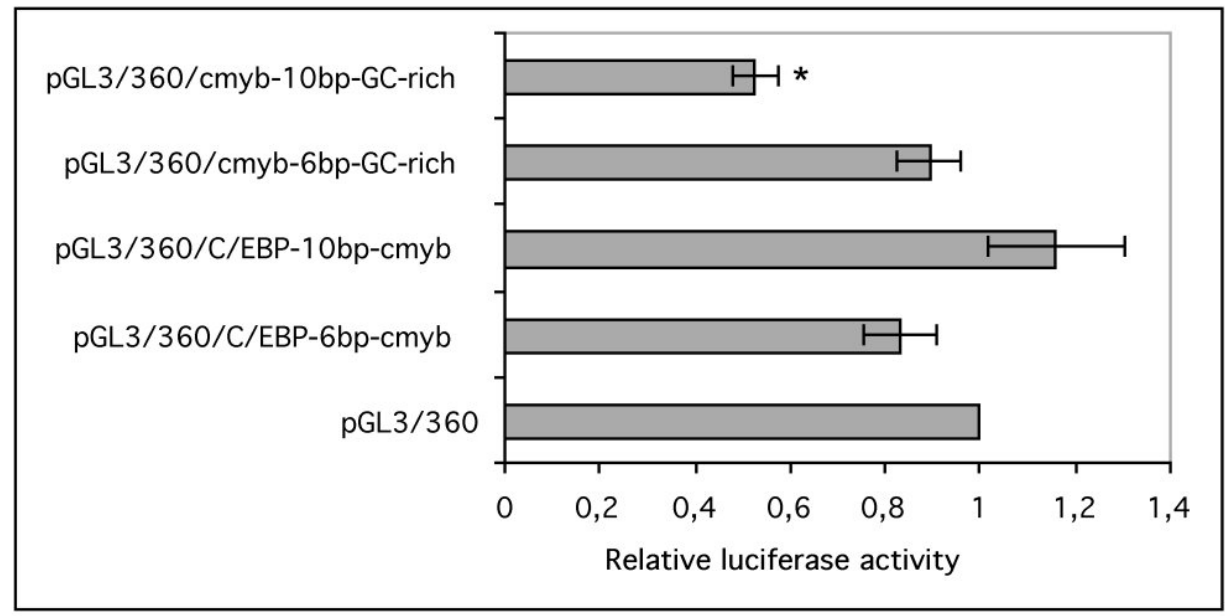

